\title{
Habitudes alimentaires du Coptodon hybride issu des croisements naturels entre Coptodon zillii (Gervais, 1848) et Coptodon guineensis (Günther, 1862) de la lagune Ebrié (Côte d'Ivoire)
}

\section{Céline Sidonie Koco NOBAH ${ }^{2}$, Kouakou Augustin KOUAME ${ }^{1 *}$, Safiatou COULIBALY ${ }^{3}$, Boua Célestin ATSE $^{3}$ et Essetchi Paul KOUAMELAN ${ }^{1}$}

${ }^{I}$ Laboratoire d'Hydrobiologie et d'Eco-Technologie des Eaux, UFR-Biosciences, Université Félix HouphouëtBoigny, 22 B.P. 582 Abidjan 22, Côte d'Ivoire.

${ }^{2}$ Ecole Normale Supérieure d'Abidjan (ENS), Département des Sciences et Technologies, Section Sciences de la Vie et de la Terre, 08 BP 10 Abidjan 08 Côte d'Ivoire.

${ }^{3}$ Centre de Recherche Océanologique d'Abidjan, Département de Génétique et Aquaculture, BP V 18 AbidjanCôte d'Ivoire.

*Auteur correspondant ; E-mail: kkouamea@yahoo.com; Tel : +22507648839.

\section{REMERCIEMENTS}

Les auteurs remercient le Fond Interprofessionnel pour la Recherche et le Conseil Agricole (FIRCA) pour avoir financé ce travail.

\section{RÉSUMÉ}

Coptodon hybride est un poisson issu des croisements naturels entre Coptodon zillii X Coptodon guineensis. La présente étude a pour objectif de caractériser les habitudes et les stratégies alimentaires de ce poisson, en fonction de la taille des individus, des stations d'échantillonnage et des saisons hydrologiques dans la lagune Ebrié (Côte d'Ivoire). Sur un total de 323 individus $(65<$ longueur standard $\leq 225 \mathrm{~mm})$ capturés d'août 2014 à juillet 2015 avec des filets maillants, 236 tubes digestifs contenaient des proies et 87 étaient vides (26,93\%). L'Indice d'Importance Relative (\%IRI) qui prend en compte les pourcentages d'occurrence, numérique et pondérale, a été utilisé pour évaluer l'importance relative des différentes catégories de proies dans les tubes digestifs. Cinq groupes de proies ont été identifiés: insectes, zooplancton, crustacés, phytoplancton et plantes terrestres. Le régime alimentaire de Coptodon hybride est essentiellement constitué de plantes terrestres, surtout de débris végétaux (91,53\% de l'IRI). L'analyse du coefficient de corrélation de Spearman montre une variation du régime en fonction de la taille et des stations. Cependant, il n'y a pas d'influences significatives des saisons sur l'alimentation des individus de petites tailles (65-129 mm) et ceux de grandes tailles (130-225 mm).

(C) 2019 International Formulae Group. All rights reserved

Mots clés: Habitudes alimentaires, Coptodon hybride, lagune Ebrié, Côte d'Ivoire.

\section{Feeding habits of hybrid Coptodon resulting from natural crosses between Coptodon zillii (Gervais, 1848) and Coptodon guineensis (Bleeker, 1862) in the Ebrié lagoon (Côte d'Ivoire)}

\author{
ABSTRACT \\ Hybrid Coptodon is a fish resulting from natural crosses between Coptodon zillii X Coptodon \\ guineensis. The objective of this study is to characterize the feeding habits of this fish, according to the size of
}


individuals, sampling sites and hydrological seasons in the Ebrié lagoon (Côte d'Ivoire). A total of 323 specimens $(65<$ standard length $\leq 225 \mathrm{~mm})$ were captured from August 2014 to July 2015 with gillnets. hundred and thirty-six digestive tracts contained prey and 87 were empty $(26.93 \%)$. The Relative Index (\%IRI), including the occurrence, numerical and weight percentages, was used to assess the importance of the different categories of prey in the digestive tract. Five groups of prey have been insects, zooplankton, crustaceans, phytoplankton and terrestrial plants. The hybrid tilapia's diet shows terrestrial plants and plant debris $(91.53 \%$ of IRI). The Spearman correlation coefficient analysis variations as a function of size and sample sites. However, there are no significant seasonal influences diet of small (65-129 mm) and large (130-225 mm) fish.

(C) 2019 International Formulae Group. All rights reserved

Keywords: Feeding habits, hybrid Coptodon, Ebrié lagoon, Côte d'Ivoire.

\section{INTRODUCTION}

La connaissance du régime et de l'éthologie alimentaires des poissons en milieu naturel est nécessaire dans la compréhension de la biologie des communautés des écosystèmes aquatiques. Ainsi, l'étude de l'alimentation peut fournir des données sur la disponibilité des proies et des facteurs bioécologiques qui pourraient influencer celle-ci, notamment la taille, l'âge, le sexe, les saisons hydrologiques et les paramètres physico-chimiques du milieu (Kouamélan et al., 2006 ; Sarr et al., 2013).

Coptodon zillii est une espèce d'eau douce (Lévêque et Paugy, 2006a) et Coptodon guineensis une espèce d'eau saumâtre (Teugels et Thys van den Audenaerde, 2003). Ces deux espèces sont morphologiquement et génétiquement apparentées et se rencontrent naturellement dans la plupart des bassins Ouest Africains (Thys van den Audenaerde, 2003). Dans les milieux subissant des perturbations écologiques, ces poissons sont sujets à une hybridation massive et incontrôlée (Nobah et al., 2006). Cependant, il existe peu de travaux sur l'écologie en général et les habitudes alimentaires de ces hybrides en particulier. Les données disponibles concernant les espèces parentales et les hybrides sont d'ordre général, fondées sur la description du spectre alimentaire de ceux-ci dans le Lac de barrage d'Ayamé (Shep et al., 2013).

Dans la lagune Ebrié, siège d'une importante activité de pêche artisanale (Bédia et al., 2009), ces Coptodons hybrides se rencontrent régulièrement dans les captures et sont très appréciés pour leur goût comme toutes les autres espèces du même genre. Ainsi, ceux-ci sont de plus en plus abondants comparativement aux espèces parentales. De ce qui précède, une étude plus approfondie du régime alimentaire portant sur l'aspect quantitatif qui est fondé sur des indices mixtes et la variation spatio-temporelle s'avère nécessaire.

Le but de ce travail est de caractériser les habitudes et les stratégies alimentaires de Coptodon hybride, en fonction de la taille des individus et de décrire l'étendue de la variabilité spatio-temporelle des Coptodons hybrides capturés dans la lagune Ébrié (Côte d'Ivoire).

\section{MATERIEL ET METHODES Milieu d'étude}

La lagune Ebrié est située au sud de la Côte d'Ivoire, avec une superficie de $566 \mathrm{~km}^{2}$, elle est la plus grande des lagunes du littoral de l'Afrique de l'Ouest (Dufour et al., 1994). Située entre les longitudes $3^{\circ} 47^{\prime}$ et $5^{\circ} 29^{\prime}$ Ouest et les latitudes $5^{\circ} 02^{\prime}$ et $5^{\circ} 42^{\prime}$ Nord, la lagune a environ $130 \mathrm{~km}$ de longueur et une profondeur moyenne de 4,8 m. Cette profondeur peut atteindre $20 \mathrm{~m}$ dans les zones près d'Abidjan (Albaret, 1994).

La lagune a été découpée en six secteurs en fonction de la production primaire et secondaire et de la pêche (Figure 1). Elle communique avec l'Océan Atlantique de façon permanente par le canal de Vridi construit en 1951, donnant ainsi des caractéristiques estuariennes typiques aux secteurs II, III et IV. Ces parties ont un gradient de salinité très marqué, allant de $0 \%$ o en saison des pluies à $30 \%$ en saison sèche. Les eaux des secteurs V et VI sont stables et homogènes (oligohalines), avec une salinité variant de $0 \%$ à $3 \%$. Elle reçoit les eaux douces du fleuve Comoé et des rivières 
Agnéby et Mé. Elle bénéficie d'un climat de type équatorial de transition incluant 4 saisons annuelles dont deux pluvieuses (avril à juillet et octobre à novembre) et deux sèches (décembre à mars et août à septembre) (Dufour et al., 1994).

\section{Echantillonnage et analyse des contenus stomacaux}

L'échantillonnage des poissons a été réalisé mensuellement d'août 2014 à juillet 2015. Ceux-ci ont été capturés au niveau des stations de Layo (secteur IV), d'Ahua (secteur V) et de Gboyo (secteur VI). Une batterie de 10 filets maillants de maille 10, 15, 20, 25, 30, $35,45,50,55$ et 60 a été utilisée. À chaque station, la batterie de filets maillants a été posée à $17 \mathrm{~h}$ et relevé le lendemain à $7 \mathrm{~h}$. Les poissons capturés ont été identifiés selon les clés de De Vos (1995), Paugy et al. (2003a et b) et Nobah et al. (2007). Les longueurs standards ont été mesurées, les estomacs prélevés et conservés dans du formol à $5 \%$. $\mathrm{Au}$ laboratoire, chaque contenu stomacal délayé dans $10 \mathrm{ml}$ d'eau, a été filtré à travers une série de tamis de $500 \mu \mathrm{m}, 250 \mu \mathrm{m}$ et 100 $\mu \mathrm{m}$ de diamètre. Les fractions ainsi obtenues et le filtrat ont été examinés respectivement à la loupe binoculaire et au microscope. Les aliments ont été triés, comptés et pesés. L'identification des insectes a été faite selon Dejoux et al. (1981) et Tachet et al. (2003). Les organismes zooplanctoniques ont été identifiés selon Pourriot et al. (1982) et Dussart $(1980 ; 1989)$. Concernant les larves de crevettes, celles-ci ont été identifiées à l'aide des clés de Monod (1966) et de N'zi (2007). Les organismes phytoplanctoniques contenus dans le filtrat ont été comptés sous un microscope entre lame et lamelle et identifiés selon Ouattara (2000) et Komoé (2010).

La contribution de chaque aliment dans le régime alimentaire a été déterminée selon différents indices proposés par Pinkas et al. (1971) :

- $\quad$ Coefficient de vacuité $(\mathrm{CV})=($ Nombre d'estomac vides / Nombre d'estomacs analysés) x 100 ;

- $\quad$ Pourcentage d'occurrence $(\% \mathrm{~F})=\left(\mathrm{N}_{\mathrm{ie}} /\right.$ $\mathrm{N}_{\mathrm{et}}$ ) x 100 , où $\mathrm{N}_{\mathrm{ie}}=$ nombre d'estomacs contenant l'item i et $\mathrm{N}_{\mathrm{et}}=$ nombre total d'estomacs pleins examinés ;

- $\quad$ Pourcentage numérique $(\% \mathrm{~N})=\left(\mathrm{N}_{\mathrm{i}} / \mathrm{N}_{\mathrm{t}}\right)$ $x 100$ où $N_{i}=$ nombre total de l'item $i$ et $\mathrm{N}_{\mathrm{t}}=$ nombre de tous les items ;

- Pourcentage pondéral $(\% \mathrm{~W})=\left(\mathrm{W}_{\mathrm{i}} / \mathrm{W}_{\mathrm{t}}\right)$ $\mathrm{x} 100$, où $\mathrm{W}_{\mathrm{i}}=$ poids total de l'item $\mathrm{i}$ et $\mathrm{W}_{\mathrm{t}}=$ poids total de tous les items ; Indice d'importance relative (IRI) $=\% \mathrm{~F}$ $\mathrm{x}(\% \mathrm{~N}+\% \mathrm{~W})$. Cet indice a été choisi en vue d'éliminer les biais occasionnés par les différents pourcentages ( $\mathrm{F}, \mathrm{N}$ et $\mathrm{W}$ ) qui le composent (Windell, 1971; Rosecchi et Nouaze, 1987). Cet indice a été exprimé en pourcentage selon la formule suivante :

$\%$ IRI $=\frac{\text { IRI }}{\sum \text { IRI }} \times 100$

$\mathrm{La}$ classification des aliments en termes d'aliments préférentiels, secondaires et accessoires a été faite selon Rosecchi et Nouaze (1987).

Les classes de tailles ont été définies sur la base de la règle de Sturge (Scherrer, 1984).

Nombre de classes $(\mathrm{NC})=1+3,3 \log _{10}$ (n) où $\mathrm{n}=$ nombre total des individus.

L'intervalle de classe (I) a été déterminé par le rapport suivant :

$\mathrm{I}=\left(\mathrm{LS}_{\max }-\mathrm{LS}_{\min }\right) / \mathrm{NC}$, où $\mathrm{LS}=$ Longueur Standard.

\section{Analyses statistiques}

Les traitements statistiques suivants ont été réalisés à l'aide du programme STATISTICA 7.1 (Statsoft inc) :

-l'analyse de classification hiérarchique ascendante pour regrouper les classes de tailles constituées de poissons aux régimes alimentaires similaires ;

-l'analyse du coefficient de corrélation des rangs de Spearman (rs) pour voir s'il existe ou non un degré de liaison entre les régimes alimentaires (probabilité critique retenue : $p=0,05)$. Si rs $=1$, les régimes sont rigoureusement identiques; si rs $=-1$, les régimes sont rigoureusement inverses. Enfin, si $\mathrm{rs}=0$, les régimes sont indépendants (Scherrer, 1984). Elle a été utilisée pour vérifier le lien entre les régimes alimentaires des classes de tailles, des stations et pendant les saisons hydrologiques. 


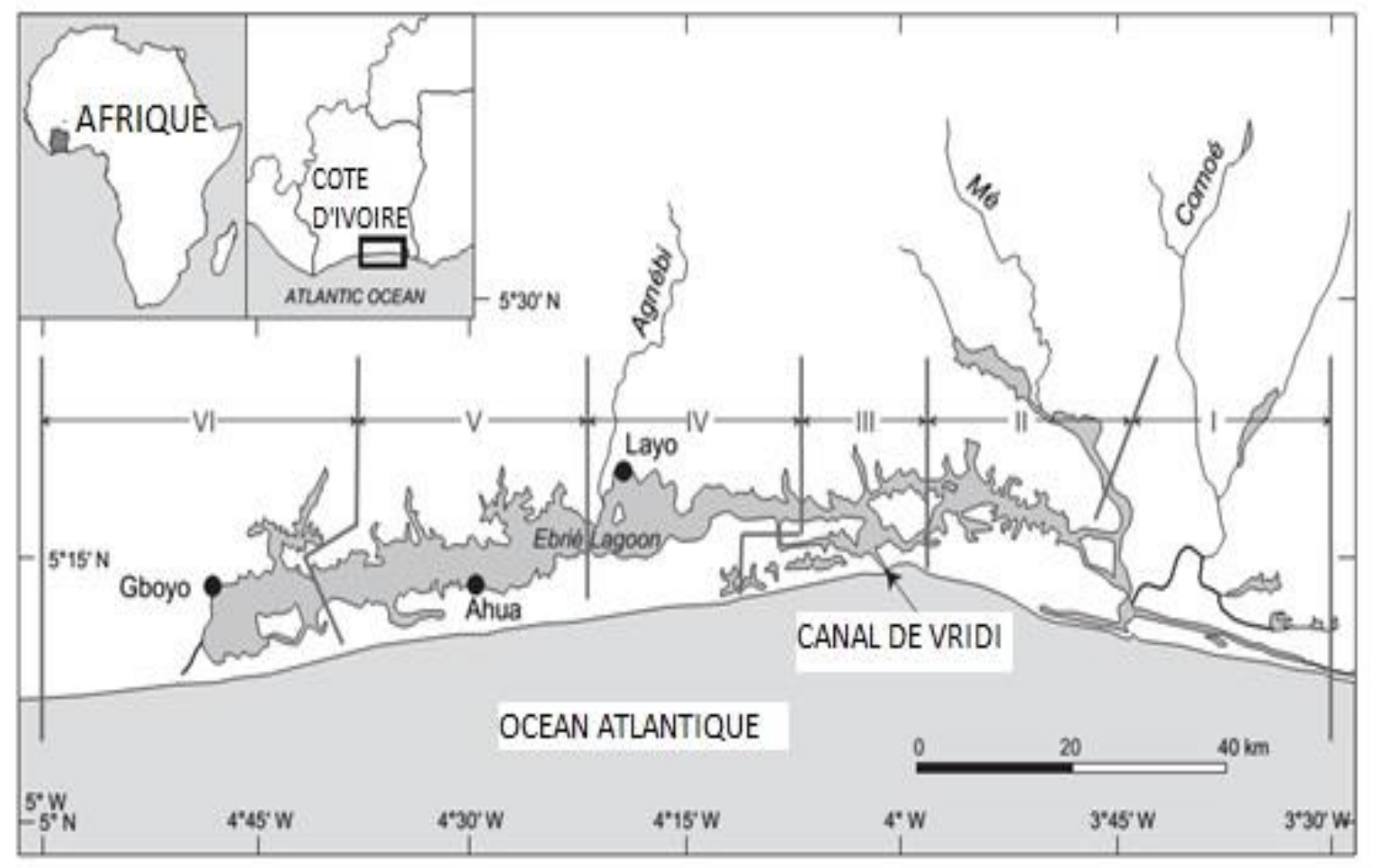

Figure 1: Situation géographique de la lagune Ébrié en Côte d'Ivoire subdivisée en secteurs I à VI et localisation des stations d'échantillonnage $(\bullet=$ station d'échantillonnage)

\section{RESULTATS}

\section{Profil général du régime alimentaire}

Pour l'ensemble des trois stations, 323 contenus digestifs de Coptodon hybride ont été examinés. Ce nombre est reparti en 236 tubes digestifs pleins et 87 vides, ce qui correspond à un pourcentage de vacuité de 26,93\%. L'analyse qualitative des différents tubes digestifs contenant des proies $(n=236)$ permet de distinguer 17 types d'aliments, regroupés en 5 catégories: insectes, zooplancton, crustacés, phytoplancton et plantes terrestres. Le régime est essentiellement constitué de plantes terrestres (93,93\% de l'IRI) comprenant 2 proies : fruits et débris végétaux (Tableau 1).

La classification des aliments à partir de leurs pourcentages de l'indice d'importance relative (IRI), permet de considérer les plantes terrestres (débris végétaux : $91,53 \%$ et fruits : $2,4 \%$ ), comme aliment préférentiel. Les insectes, secondairement consommés, constituent $6,03 \%$ de la nourriture avec une proportion de Diptères de $5,15 \%$ de l'IRI. Les autres groupes taxonomiques: zooplancton, crustacés et phytoplancton présentent des valeurs d'IRI les plus faibles $(<5 \%)$, indiquant que ce sont des aliments accidentels dans le régime alimentaire de Coptodon hybride.

\section{Variation du régime alimentaire en} fonction de la taille des individus

Les poissons étudiés ont des longueurs standards comprises entre $65 \mathrm{~mm}$ et $225 \mathrm{~mm}$. A partir de la règle de Sturge, 10 classes de tailles (16 mm d'intervalle) ont été établies. En raison des faibles effectifs, les deux dernières classes ont été fusionnées en une 
seule. En conséquence, 9 classes ont pu être constituées (Tableau 2).

Le dendrogramme obtenu à partir des différentes classes de tailles indique deux groupes (Figure 2). Le groupe 1 renferme tous les individus de tailles inférieures à $129 \mathrm{~mm}$ $(\mathrm{n}=95)$, c'est-à-dire les quatre premières classes de tailles, et le groupe 2 contient ceux dont la taille est supérieure à $129 \mathrm{~mm}(\mathrm{n}=$ 228), soit les individus des cinq dernières classes.

L'indice d'importance relative des différents items-proies a été calculé pour chaque groupe (Figure 3). Chez les individus du groupe 1 , les insectes $(73,60 \%)$ et le zooplancton $\quad(17,38 \%) \quad$ constituent respectivement les aliments préférentiels et secondaires. En revanche, chez les individus du groupe 2, le régime alimentaire est dominé par les plantes terrestres $(78,18 \%)$ et les insectes qui constituent des proies secondaires $(15,92 \%)$.

Le test de corrélation de rang de Spearman effectué entre les individus des deux groupes indique qu'il n'y a pas de similitude entre les régimes alimentaires des poissons (Tableau 3).

\section{Variation du régime alimentaire en fonction des stations}

La composition des aliments consommés par les Coptodons hybrides en fonction des stations ainsi que leur indice d'importance relative sont consignés dans le Tableau 3. Au total, 17 types de proies ont été identifiés dans chacune des trois stations. Les plantes terrestres (fruits + débris végétaux) sont les aliments consommés préférentiellement dans toutes les stations. Les indices d'importance relative sont de $96,89 \%$, $95,89 \%$ et $95,06 \%$ respectivement à Layo (secteur IV), Ahua (secteur V) et Gboyo (secteur VI). Toutefois, Chironomus sp. est classée comme un aliment principal à Layo (30\%) tandis qu'il est consommé de manière accidentelle à Ahua et Gboyo (IRI < 5\%).

Le test de corrélation de rang de Spearman effectué entre les stations prises deux à deux indique qu'il y a similitude entre les régimes alimentaires des Coptodons hybrides des couples de stations Layo-Ahua et Ahua-Gboyo. En revanche, les régimes ne sont pas similaires pour le couple LayoGboyo (Tableau 3).

\section{Variation du régime alimentaire en fonction des saisons hydrologiques}

La composition du régime alimentaire des spécimens des deux groupes a été analysée en fonction des grandes saisons hydrologiques (Figure 4).

Chez les individus du groupe 1, quelle que soit la saison, les insectes représentent les aliments préférentiels $(72,90 \%$ en saison sèche et $78,15 \%$ en saison des pluies). Le zooplancton $(18,08 \%$ et $11,06 \%)$ constitue les aliments secondaires (en saison sèche et en saison des pluies respectivement). Les individus $\mathrm{du}$ groupe 2 consomment essentiellement les plantes terrestres $(78,55 \%$ en saison sèche et $86,18 \%$ en saison des pluies). Chez ces derniers, les insectes $(11,10 \%$ et $14,05 \%) \quad$ constituent respectivement les aliments secondaires en saison sèche et en saison des pluies.

Les coefficients de corrélation de rang de Spearman ont été calculés en fonctions des saisons hydrologiques chez les deux groupes d'individus. Les résultats indiquent qu'il y a similitude entre les régimes alimentaires des poissons quelle que soit le groupe (Tableau 4). 
Tableau 1 : Composition du régime alimentaire de Coptodon hybride dans la lagune Ébrié (Côte d'Ivoire).

\begin{tabular}{|c|c|c|c|c|}
\hline ALIMENTS & $\% \mathbf{N}$ & $\% \mathrm{~W}$ & $\% \mathbf{F}$ & $\%$ IRI \\
\hline \multicolumn{5}{|l|}{ Insectes } \\
\hline \multicolumn{5}{|l|}{ Diptères } \\
\hline Chaoborus sp. & 2,50 & 0,10 & 0,30 & 0,01 \\
\hline Chironomus sp. & 18,30 & 13,10 & 14,10 & 5,12 \\
\hline Polypedilum sp. & 3,70 & 0,10 & 0,50 & 0,02 \\
\hline \multicolumn{5}{|l|}{ Tricoptères } \\
\hline Ecnomus sp. & 0,20 & 0,10 & 0,30 & 0,00 \\
\hline Hydropsychidae & 0,10 & 0,10 & 0,20 & 0,00 \\
\hline \multicolumn{5}{|l|}{ Epheméroptères } \\
\hline Cloeon sp. & 0,10 & 0,10 & 0,20 & 0,00 \\
\hline Indéterminés & 0,30 & 0,20 & 0,30 & 0,00 \\
\hline Débris d'insectes & 4,10 & 6,60 & 7,10 & 0,88 \\
\hline \multicolumn{5}{|l|}{ Zooplancton } \\
\hline \multicolumn{5}{|l|}{ Rotifères } \\
\hline Brachionus sp. & 0,30 & 0,00 & 0,10 & 0,00 \\
\hline Keratella sp. & 0,20 & 0,00 & 0,10 & 0,00 \\
\hline \multicolumn{5}{|l|}{ Copépodes } \\
\hline Thermocyclops sp. & 0,10 & 0,00 & 1,10 & 0,00 \\
\hline \multicolumn{5}{|l|}{ Cladocères } \\
\hline Cerodaphnia sp. & 0,10 & 0,00 & 0,20 & 0,00 \\
\hline \multicolumn{5}{|l|}{ Crustaces } \\
\hline Macrobrachium sp. & 0,50 & 2,10 & 0,40 & 0,01 \\
\hline Penaeus notialis & 0,30 & 2,50 & 0,60 & 0,02 \\
\hline Phytoplancton & 0,10 & 0,00 & 0,30 & 0,00 \\
\hline \multicolumn{5}{|l|}{ Plantes terrestres } \\
\hline Fruits & 9,08 & 11,50 & 10,10 & 2,40 \\
\hline Débris végétaux & 60,02 & 63,50 & 64,10 & 91,53 \\
\hline \multicolumn{5}{|l|}{ TOTAL } \\
\hline Insectes & 29,30 & 20,40 & 23,00 & 6,03 \\
\hline Zooplancton & 0,70 & 0,00 & 1,50 & 0,00 \\
\hline Crustacés & 0,80 & 4,60 & 1,00 & 0,03 \\
\hline Phytoplancton & 0,10 & 0,00 & 0,30 & 0,00 \\
\hline Plantes terrestres & 69,10 & 75,00 & 74,20 & 93,93 \\
\hline
\end{tabular}

$\mathrm{N}=$ Pourcentage numérique ; $\mathrm{W}=$ Pourcentage pondéral ; $\mathrm{F}=$ Pourcentage d'occurrence ; IRI = Indice d'importance relative. 
Tableau 2 : Différentes classes de tailles de Coptodon hybride obtenues par la règle de Sturge et leurs effectifs.

\begin{tabular}{lll}
\hline Classe & Etendue $(\mathbf{L S}$ en $\mathbf{~ m m})$ & Effectif \\
\hline 1 & {$[65 ; 81[$} & 25 \\
2 & {$[81 ; 97[$} & 27 \\
3 & {$[97 ; 113[$} & 11 \\
4 & {$[113 ; 129[$} & 32 \\
5 & {$[129 ; 145[$} & 74 \\
6 & {$[145 ; 161[$} & 91 \\
7 & {$[161 ; 177[$} & 39 \\
8 & {$[177 ; 193[$} & 15 \\
9 & {$[193 ; 225[$} & 9 \\
\hline
\end{tabular}

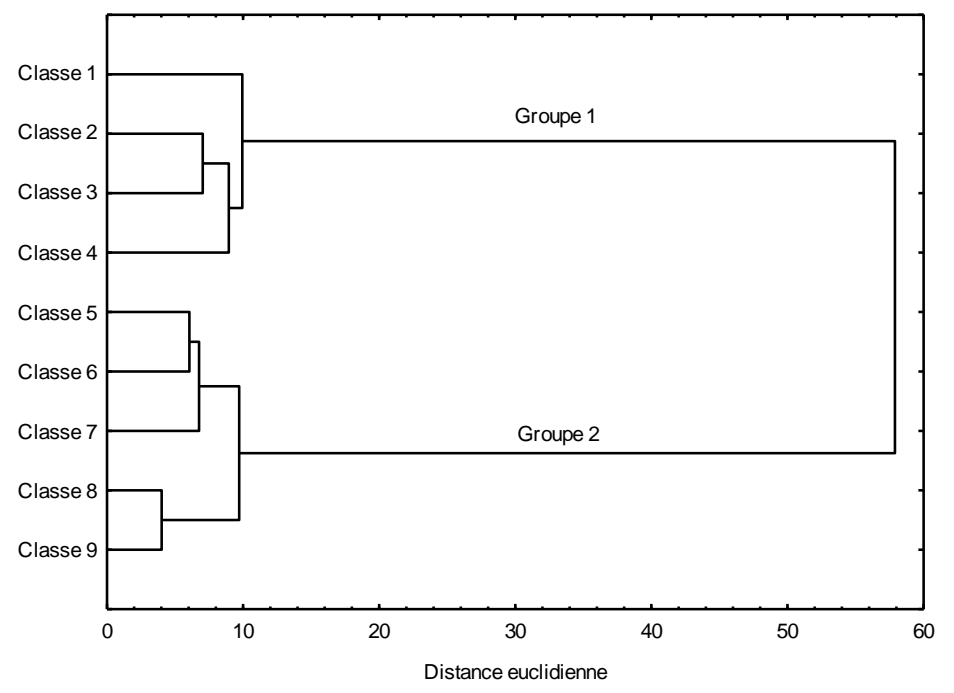

Figure 2 : Dendrogramme montrant les similarités alimentaires entre les échantillons de neuf classes de tailles de Coptodon hybride de la lagune Ébrié (Côte d'ivoire). Analyse effectuée sur la base de la distance euclidienne et utilisant la méthode de Ward. 


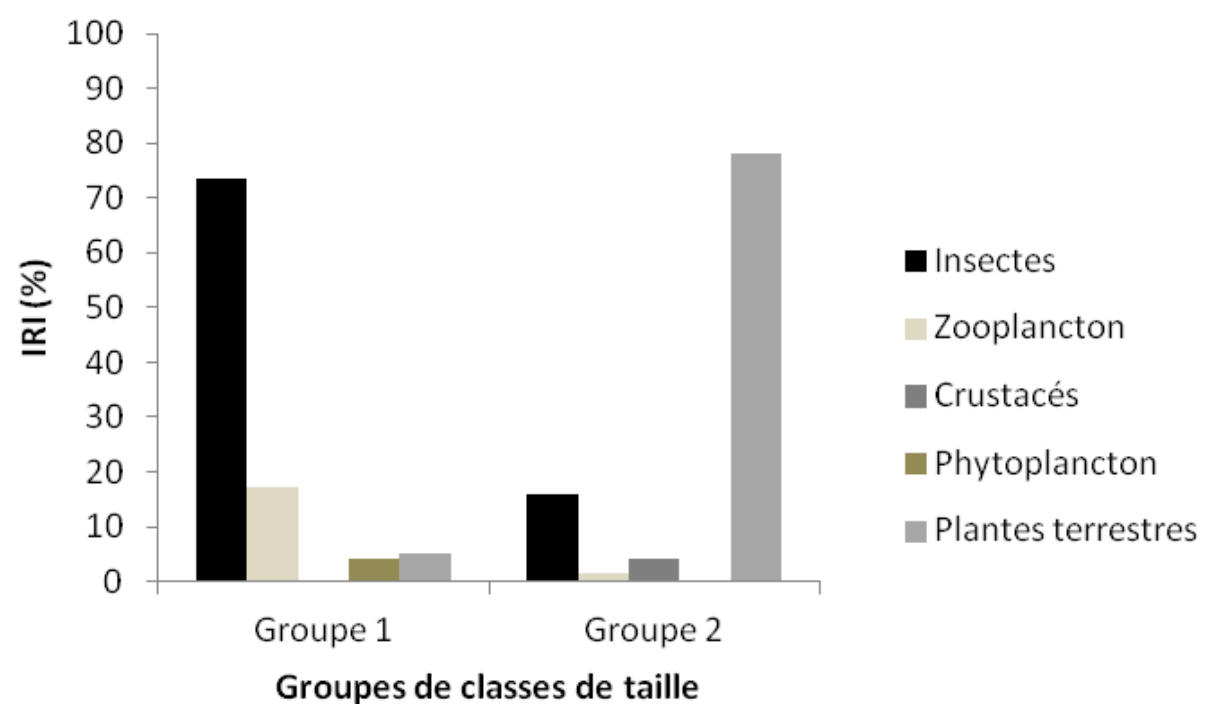

Figure 3 : Variation du régime alimentaire (IRI) de Coptodon hybride en fonction de la taille. [Groupe $1(\mathrm{n}=95)=\mathrm{LS}<129 \mathrm{~mm}$; Groupe $2(\mathrm{n}=228)=\mathrm{LS} \geq 129 \mathrm{~mm}$ ]. LS = Longueur Standard.

Tableau 3: Résultats de l'analyse de corrélation de rangs de Spearman comparant les régimes alimentaires de Coptodon hybride en fonction des classes de taille (CT), des sites d'échantillonnage et des saisons hydrologiques dans la lagune Ebrié (Côte d'Ivoire).

\begin{tabular}{llll}
\hline Source de variation & $\begin{array}{l}\text { Coefficient de t }(N-2) \\
\text { corrélation de } \\
\text { rangs de Spearman }\end{array}$ & \\
\hline
\end{tabular}

\section{Classes de tailles}

Groupe1[CT1-CT2-CT3-CT4]-Groupe2 $\quad 0,260$ [CT5-CT6- CT7-CT8-CT9]

$1,043 \quad 0,314$

\section{Sites d'échantillonnage}

$\begin{array}{llll}\text { Layo-Ahua } & 0,998 & 55,182 & 0,000001^{*} \\ \text { Ahua-Gboyo } & 0,555 & 2,587 & 0,021^{*} \\ \text { Layo-Gboyo } & 0,340 & 1,399 & 0,182 \\ \text { Saisons hydrologiques } & & & \\ \text { Groupe 1 (ss)-Groupe 1 (sp) } & 0,602 & 3,016 & 0,008^{*} \\ \text { Groupe 2 (ss)-Groupe 2 (sp) } & 0,886 & 7,663 & 0,000001^{*}\end{array}$

Groupe $1[\mathrm{CT} 1=65<\mathrm{LS} \leq 85 \mathrm{~mm} ; \mathrm{CT} 2=81<\mathrm{LS} \leq 97 \mathrm{~mm} ; \mathrm{CT} 3=97<\mathrm{LS} \leq 113 \mathrm{~mm} ; \mathrm{CT} 4=113<\mathrm{LS} \leq 129 \mathrm{~mm}]$ Groupe 2 [CT5 $=129<\mathrm{LS} \leq 145 \mathrm{~mm} ; \mathrm{CT} 6=145<\mathrm{LS} \leq 161 \mathrm{~mm} ; \mathrm{CT} 7=161<\mathrm{LS} \leq 177 \mathrm{~mm} ; \mathrm{CT} 8=177<\mathrm{LS} \leq 193 \mathrm{~mm}$; CT $9=193<$ LS $\leq 225 \mathrm{~mm}]$

$\mathrm{ss}=$ saison sèche $; \mathrm{sp}=$ saison pluvieuse $; *=$ corrélation significative à $p<0,05$.

LS = Longueur Standard 


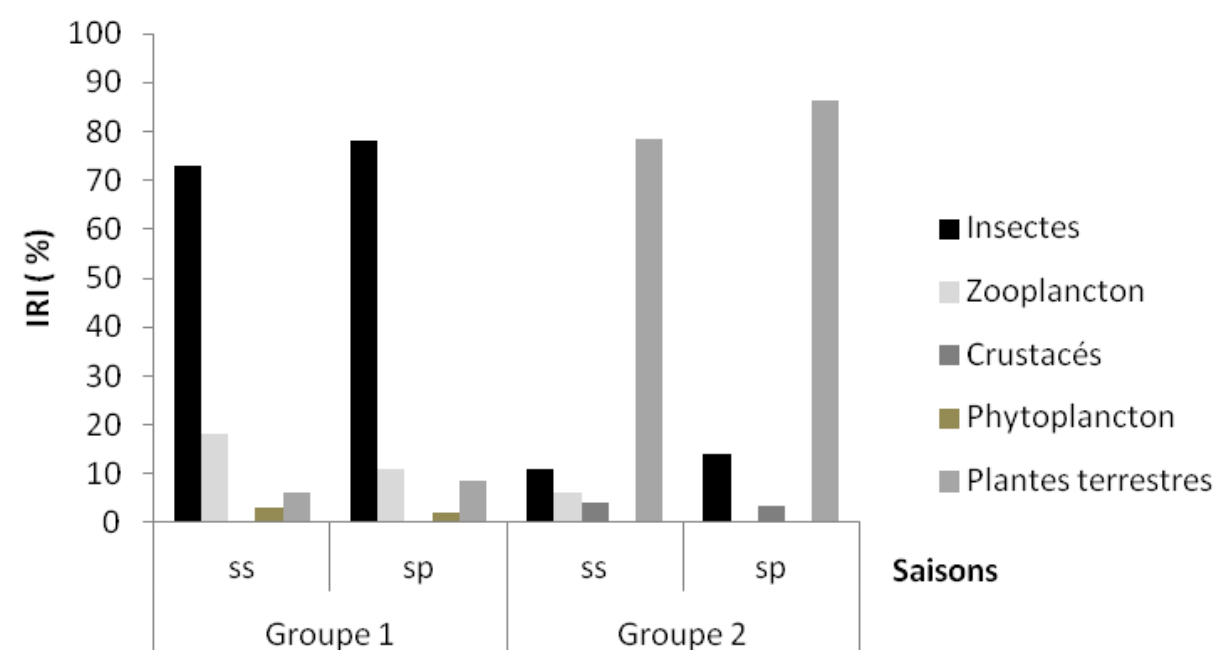

Figure 4 : Régime alimentaire de Coptodon hybride en fonction des grandes saisons hydrologiques. ( $\mathrm{ss}=$ saison sèche $; \mathrm{sp}=$ saison des pluies).

Tableau 4: Variation du régime alimentaire de Coptodon hybride en fonction des stations.

\begin{tabular}{|c|c|c|c|}
\hline \multicolumn{4}{|c|}{ STATIONS (\% IRI) } \\
\hline ALIMENTS & Layo $(\mathbf{n}=90)$ & Ahua $(n=81)$ & Gboyo $(n=65)$ \\
\hline \multicolumn{4}{|l|}{ Insectes } \\
\hline \multicolumn{4}{|l|}{ Diptères } \\
\hline Chaoborus sp. & 0,00 & 0,00 & 0,30 \\
\hline Chironomus sp. & 30,00 & 4,00 & 0,24 \\
\hline Polypedilum sp. & 0,03 & 0,03 & 0,63 \\
\hline \multicolumn{4}{|l|}{ Tricoptères } \\
\hline Ecnomus sp. & 0,01 & 0,01 & 0,58 \\
\hline Hydropsychidae & 0,01 & 0,01 & 1,17 \\
\hline \multicolumn{4}{|l|}{ Epheméroptères } \\
\hline Cloeon sp. & 0,01 & 0,01 & 0,24 \\
\hline Indéterminés & 0,01 & 0,01 & 0,34 \\
\hline Débris d'insectes & 0,00 & 0,00 & 0,06 \\
\hline \multicolumn{4}{|l|}{ Zooplancton } \\
\hline \multicolumn{4}{|l|}{ Rotifères } \\
\hline Brachionus sp. & 0,03 & 0,03 & 0,12 \\
\hline Keratella sp. & 0,00 & 0,00 & 0,92 \\
\hline \multicolumn{4}{|l|}{ Copépodes } \\
\hline Thermocyclops sp. & 0,01 & 0,01 & 0,08 \\
\hline \multicolumn{4}{|l|}{ Cladocères } \\
\hline Cerodaphnia sp. & 0,00 & 0,00 & 0,28 \\
\hline & 3315 & & \\
\hline
\end{tabular}




\section{Crustacés}

\begin{tabular}{llll}
\multicolumn{1}{l}{ Macrobrachium sp. } & 0,00 & 0,00 & 0,00 \\
$\quad$ Penaeus notialis & 0,01 & 0,01 & 0,00 \\
Phytoplancton & 0,00 & 0,00 & 0,00 \\
$\begin{array}{l}\text { Plantes terrestres } \\
\quad \text { Fruits }\end{array}$ & & \\
$\quad 13,09$ & 20,09 & 17,00 \\
$\quad$ Débris végétaux & 56,80 & 75,80 & 78,06 \\
\hline TOTAL & & & \\
Insectes & 30,05 & 3,55 & 4,05 \\
Zooplancton & 0,04 & 0,04 & 1,39 \\
Crustacés & 0,01 & 0,01 & 0,00 \\
Phytoplancton & 0,00 & 0,00 & 0,00 \\
Plantes terrestres & 69,89 & 95,89 & 95,06 \\
\hline \multicolumn{1}{l}{ IRI: Indice d'importance relative; $\mathrm{n}:$ nombre de tubes digestifs examinés } \\
\end{tabular}

\section{DISCUSSION}

Le spectre alimentaire des Coptodons hybrides dans les secteurs IV, V et VI de la lagune Ebrié est étendu; il est composé d'insectes, de zooplancton, de crustacés, de phytoplancton et de plantes terrestres. Cependant, cette espèce se nourrit principalement de plantes terrestres (débris végétaux et fruits) et secondairement d'insectes. Les résultats obtenus corroborent ceux de Shep et al. (2013), qui soulignent que le régime alimentaire des Coptodons hybrides est surtout constitué de macrophytes et d'insectes (Diptères). La présence de sable dans leurs contenus stomacaux est due à une prise accidentelle lors de la capture de proies sur le substrat (larves de Chironomidae, Chaoborus sp. etc). Ce sédiment n'intervient pas dans l'alimentation mais plutôt dans la digestion mécanique c'est à dire le déchiquetage de l'exosquelette des aliments (Kouamélan et al., 2006).

Un large spectre alimentaire a été aussi bien observé chez les Coptodons hybrides que chez les espèces parentales Coptodon zillii et C. guineensis dans le lac de barrage d'Ayamé (Shep et al., 2013). Cette diversité des catégories de proies indique un opportunisme alimentaire en fonction des disponibilités du milieu, conférant à l'espèce parentale
Coptodon zillii un régime omnivore dans la rivière Otamiri au Nigéria (Agbabiaka, 2012).

Toutefois, l'importance quantitative des deux principales proies (plantes terrestres et insectes) varie selon la taille des individus. En effet, les individus de petites tailles (groupe 1) consomment principalement les insectes et secondairement le zooplancton. En revanche, les individus de grande taille (groupe 2) ont un régime essentiellement composé d'aliments exogènes (débris végétaux et fruits). Cette différence pourrait s'expliquer par une stratégie alimentaire visant à réduire la compétition intraspécifique, offrant ainsi un large spectre alimentaire exploitable aux individus appartenant à la même espèce (Herder et Freyhof, 2016). Ainsi, les habitudes alimentaires des poissons sont souvent corrélées aux changements ontogénétiques, impliquant presque toujours le choix des proies de grandes tailles pouvant fournir une grande quantité d'énergie nécessaire à la croissance et à la reproduction (Castillo-Rivera, 2013). Pour la couverture de ces besoins énergétiques, la recherche et la collecte des proies de petite taille par les individus de grande taille s'avèrent plus coûteuse que celle de grande taille (Paugy et Lévêque, 2006b).

L'analyse du régime alimentaire des Coptodons hybrides en fonction de l'espace 
montre que les plantes terrestres à savoir les débris végétaux et les fruits constituent les proies essentielles dans toutes les stations. En effet, ces quantités appréciables dans les tubes digestifs s'expliqueraient par le débouché en lagune de la rivière Agnébi qui regorgerait une quantité importante de fruits et de débris végétaux issus du ruissellement après la pluie et du transport par les vents (Doumbia, 2003). Les débris végétaux consommés seraient des jeunes pousses que les poissons broutent en surface (Konan et al., 2008).

Une analyse approfondie des résultats a mis en évidence une variation du régime alimentaire en fonction des stations qui sont pourtant très proches géographiquement. En effet, l'importance relative de Chironomus sp. comme aliment principal dans le régime alimentaire des Coptodons hybrides à Layo (secteur IV) serait due à une abondance de Chironomidae dans les zones benthiques de la rivière Agnébi qui débouche sur cette station (Diomandé, 2001). Ces observations sont conformes à celles de Appia et al. (2017), qui indiquent que les Chironomidae sont les plus abondants à la station de Layo, constituant ainsi une source trophique très accessible pour les individus des deux groupes de classes de tailles. De plus, la baisse de la salinité due aux facteurs tels que les précipitations et aux crues du fleuve Comoé et de la rivière Agnébi, créeraient des conditions physico-chimiques adéquates à la prolifération des insectes notamment des Chironomidae, ce qui augmenterait leur nombre dans l'alimentation de Coptodon hybride.

Par ailleurs, les résultats de la variation saisonnière du régime alimentaire au sein de chaque groupe montrent qu'il n'y a pas de changement significatif. Toutefois, il y a une augmentation plus ou moins considérable de la part des insectes (groupe 1) et des plantes terrestres (groupe 2) en saison des pluies. Le nombre élevé de catégories d'aliments enregistré en saison pluvieuse pourrait être attribué à la remontée des eaux. Ainsi, cette légère variation du régime alimentaire en fonction des saisons serait liée à la disponibilité des proies durant les saisons pluvieuses. En effet, durant ces saisons, le ruissellement des eaux apporte aux milieux aquatiques une quantité importante de matière organique (matières végétales, insectes et nutriments) d'origine terrestre qui vient enrichir ces milieux (Castillo-Rivera, 2013).

\section{Conclusion}

L'étude des habitudes alimentaires de Coptodon hybride (Coptodon zillii X Coptodon guineensis) en fonction de la taille des individus, des stations d'échantillonnage et des saisons hydrologiques dans la lagune Ebrié montre qu'il a un spectre alimentaire diversifié, dû à la disponibilité et à l'abondance des proies dans le milieu. En effet, la forte consommation d'aliments exogènes (débris végétaux et fruits) montre qu'il s'agit d'une espèce qui appartient au groupe des consommateurs primaires. Cependant, quelle que soit la station d'étude et la saison d'échantillonnage, l'espèce peut toujours être classée parmi les omnivores à tendance macrophytophage.

Les secteurs IV, V et VI de la lagune Ebrié devraient faire l'objet d'une étude plus approfondie de l'impact des activités anthropiques sur la qualité de l'eau, ce qui permettrait de proposer un programme de gestion efficace de cette lagune.

\section{CONFLIT D'INTERETS}

Les auteurs de cet article déclarent qu'il n'y a aucun conflit d'intérêts dans cette publication.

\section{CONTRIBUTIONS DES AUTEURS}

$\mathrm{Ce}$ manuscrit porte sur un projet de recherche co-rédigé et co-exécuté par CSKN, $\mathrm{SC}$ et BCA sous la supervision de EPK. Les autres auteurs du manuscrit sont CSKN (pour la rédaction du manuscrit), KAK (pour le traitement statistique des données et la détermination scientifique des noms des proies) et SC (pour avoir contribué à la recherche bibliographique et la lecture du manuscrit).

\section{REMERCIEMENTS}

Ce travail a été réalisé dans le cadre d'un projet de recherche intitulé «Impact des changements environnementaux sur la vulnérabilité des ressources halieutiques dans la lagune Ebrié (Côte d'Ivoire) ». Il a bénéficié des financements du Fond 
Interprofessionnel pour la Recherche et le Conseil Agricole (FIRCA) en collaboration avec le Centre de Recherches Océanologiques (C.R.O.) d'Abidjan et le Ministère de l'Agriculture et des Ressources Halieutiques.

\section{REFERENCES}

Agbabiaka LA. 2012. Food and feeding habits of Tilapia zillii (Pisces: Cichlidae) in river Otamiri South-eastern, Nigeria. Biosci. Disc., 3 (2): 146-148.

Albaret JJ. 1994. Les poissons : biologie et peuplements. In Environnement et Ressources Aquatiques de Côte d'ivoire (Tome II). Les Milieux Lagunaires, Durand JR, Dufour P, Guiral D, Zabi SG (eds). Orstom : Paris; 239-279.

Appia YS, Etilé RN, Kouamé KA, Kouamelan EP. 2017. Benthic macroinvertebrates composition and spatiotemporal Variation in relationship with environmental parameters in a coastal tropical lagoon (Ebrié Lagoon, Côte D'ivoire). Int. J. Sci. Res. Meth., 7 (4): 149-169.

Bedia AT, N'zi KG, Yao SS, Kouamélan EP, N'douba V, Kouassi NJ. 2009. Typologie de la pêche en lagune AghienPotou (Côte d'Ivoire, Afrique de l'ouest): acteurs et engins de pêche. Agron. Afr., 21 (2): 197-204. DOI: http://dx.doi.org/10.4314/aga.v21i2.4981 0

Castillo-Rivera M. 2013. Influence of rainfall pattern in the seasonal variation of fish abundance in a tropical estuary with restricted marine communication. $J$. W. Res. Protec., 5: 311-319. DOI : http://dx.doi.org/10.4236/jwarp.2013.53 A032.

Dejoux C, Elouard JM, Forge P, Maslin JL. 1981. Catalogue iconographique des insectes aquatiques de Côte d'Ivoire. Rapport ORSTOM 42.

De Vos L. 1995. A systematic revision of the African Schilbeidae (Teleostei, Siluriformes), with an annotated bibliography. Mus. R. Afr. Centr., An. Sci. Zoo., 271: 1-450.

Diomande D, Gourene G, Tito de Morais. 2001. Stratégies alimentaires de Synodontis bastiani (Siluriformes,
Mochokidae) dans le complexe fluviolacustre de la Bia. Cybium, 25 (1) : 7-21.

Doumbia L. 2003. Variation spatio-temporelle des peuplements et stratégies alimentaires de deux poissons-chats africains : Schilbe mandibularis (Günther, 1867) et Schilbe intermedius (Rüppel, 1832) (Bassins Bia et agnébi ; Côte d'ivoire). Thèse de doctorat, Univ. Abobo-Adjamé, Côte d'ivoire, p.189.

Dufour P, Kouassi AM, Lanusse A. 1994. Les populations. In Environnement et Ressources Aquatiques de Côte d'ivoire. (Tome II). Les Milieux Lagunaires, Durand JR, Dufour P, GUIRAL D, Zabi SG (eds). ORSTOM : Paris ; 309-333.

Dussart BH. 1980. Copépodes. In Flore et Faune Aquatiques de l'Afrique Sahélosoudanienne, Durand JR, Lévêque C (eds). ORSTOM: Paris ; 333-356.

Dussart BH. 1989. Crustacés Copépodes Calanoïdes des eaux intérieures africaines. Int. J. Crust. Res., 15: 1-205.

Herder F, Freyhof J. 2006. Ressource partitioning in a tropical stream fish assemblage. J. Fish Bio., 69 : 571-589. DOI:10.1111/j.1095-8649.2006.01126.x

Komoé K. 2010. Distribution du phytoplancton dans le complexe lagunaire de Grand Lahou en Côte d'Ivoire. Thèse de Doctorat, Université de Cocody-Abidjan, p. 282.

Konan KJ, Atsé BC, Kouassi NJ. 2008. Habitudes et stratégies alimentaires de Tylochromis jentinki jentinki (Cichlidae) dans la lagune Ébrié (Côte d'Ivoire). Cybium, 32 (1): 3-8.

Kouamélan EP, Koné T, N'Douba V, Oliver F. 2006. Food habits and trophic resource partitioning among three African mormyrid fishes from man-made lake Ayamé (Ivory Coast). Afr. Zool., 41: 266-274. DOI : https://doi.org/10.1080/ 15627020.2006. 11407362

Monod T. 1966. Crevettes et crabes des côtes occidentales de l'Afrique. Réunion des spécialistes C.S.A sur les crustacées, Zanzibar, 1964. Mém. Inst. Fond. Afr. Noire : 106-234.

Nobah CSK, Koné T, N'Douba V, Snoeks J, Gooré Bi G, Kouamelan EP. 2006. Comparative analysis of the aquaculture 
potential of hybrid Tilapia zillii (male) $\mathrm{x}$ T. guineensis (female) (Teleostei: Cichlidae) in floating cages, cement tanks and earth ponds. Afr. J. Aqu. Sci., 31 (2): 319-323. DOI : https://doi.org/10.2989/160859 10609503902

Nobah CSK. 2007. Critères d'identification et performances zootechniques des Tilapias hybrides [Tilapia zillii (Gervais, 1948) X T. guineensis (Günther, 1862)] dans trois structures d'élevage: cages flottantes (lac d'ayamé), étangs en terre et bassins en béton (Aboisso). Thèse de doctorat de l'Université de Cocody-Abidjan, Côte d'Ivoire, p. 220.

N'Zi KG. 2007. Diversité biologique des peuplements de crevettes des eaux douces de Côte d'Ivoire en relation avec les variables environnementales. Thèse de Doctorat. Université de CocodyAbidjan, Côte d'Ivoire, p. 178.

Ouattara A. 2000. Premières données systématiques et écologiques du phytoplancton du lac d'Ayamé (Côte d'Ivoire). Thèse de Doctorat. Katholieke Universiteit Leuven, Belgique, p. 207.

Paugy D, Lévêque C. 2006a. Taxinomie et systématique. In Les Poissons des Eaux Continentales Africaines : Diversité, Ecologie, Utilisation par l'Homme, Lévêque C, Paugy D (eds). Editions de l'IRD : Paris ; 105-134.

Paugy D, Lévêque C. 2006b. Régime alimentaire et réseaux trophiques. In Les Poissons des Eaux Continentales Africaines : Diversité, Ecologie, Utilisation par l'Homme, Lévêque C, Paugy D (eds). Editions de l'IRD: Paris ; 191-216.

Paugy D, Lévêque C, Teugels GG. 2003a. Poissons d'Eaux Douces et Saumâtres de l'Afrique de l'Ouest (Tome 1). IRD Éditions, Collection Faune et Flore Tropicales ; $457 \mathrm{p}$.

Paugy D, Lévêque C, Teugels GG. 2003b. Poissons d'Eaux Douces et Saumâtres de l'Afrique de l'Ouest (Tome 2). IRD Editions, Collection Faune et Flore Tropicales, $815 \mathrm{p}$.
Pinkas L, Oliphant MS, Iverson ILK. 1971. Food habits of albacore, blue fin tuna and bonito in Californian waters. Californie Fish Game, 152: 1-105.

Pourriot R, Capblancq J, Champs P, Meyer JC. 1982. Écologie du Plancton des Eaux Continentales. Masson: Paris ; 198p.

Rosecchi E, Nouaze Y. 1987. Comparaison de cinq indices utilisés dans l'analyse des contenus stomacaux. Rev. Trav. Inst. Pêche Marit., 49: 11-123.

Sarr SM, Kabre JT, Niass F. 2013. Régime alimentaire du mulet jaune (Mugil cephalus, Linneaus, 1758, Mugilidea) dans l'estuaire du fleuve Sénégal. J. App. Bios., 71: 5663-5672. DOI : http://dx.doi.org/10.4314/jab.v71i1.9880 9

Scherrer B. 1984. Résultat des données. In Biostatistique. Gaetan Morin éditeur: Louiseville, Canada.

Shep H, Konan KM, Doumbia L, Ouattara M, Boussou KC, Ouattara A, Gourène G. 2013. Feeding Relationships Among Tilapia zillii (Gervais, 1848), Tilapia guineensis (Günther, 1862) and Their Hybrid in Ayamé Man-Made Lake, Côte d'Ivoire. Pakistan J. Zoo., 45(5) : 14051414.

Tachet H, Richoux P, Bournaud M, Usseglio Polatera P. 2003. Invertébrés d'Eau Douce : Systématique, Biologie et Écologie. CNRS : Lyon.

Teugels GG, Thys Van Den Audenaerde DFE. 2003. Les Cichlidae. In Poissons d'Eaux Douces et Saumâtres de l'Afrique de L'Ouest, Paugy D, Lévêque C, Teugels GG. (eds). IRDMNHN/MRAC : Paris et Tervuren ; 520-599.

Thys Van Den Audenaerde DFE, Teugels GG. 2003. Les Cichlidae. In Poissons d'Eaux Douces et Saumâtres de l'Afrique de L'Ouest, Paugy D, Lévêque C, Teugels GG (eds). IRDMNHN/MRAC : Paris et Tervuren; 520-599.

Windellj T. 1971. Food analysis and rate of digestion. In Methods for Assessment of Fish Production in Freshwater, Ricker WE (ed). Oxford: Blackwell Scientific Publications; 215-226. 